\title{
Fascia and living tensegrity considerations in lower extremity and pelvic entrapment neuropathies
}

\author{
John Sharkey.
}

Faculty of Medicine, Dentistry and Clinical Sciences, University of Chester/NTC, 15-16aSt Joseph's Parade, Dorset St, DO7 FR6C, Dublin, Ireland.

\section{ABSTRACT}

Peripheral neuropathies can have a plethora of origins including physical insults resulting from connective tissue compression and entrapment. Observational investigations, using biotensegrity focused dissections, have identified site-specific fascial structures that are hypothesised to afford integrity to neurovascular structuresby providing appropriate tension and compression. These myofascial structures act as site-specific fascia tuning pegs. While these 'tuning pegs' are capable of having a whole body impact, this paper will look specifically at the local influences on pelvis and lower limb. The analogy of a fascia 'tuning peg', similar to the tuning peg of a string instrument, is adoptedto help explain this unfamiliar concept. An 'out of tune' fascial system would lead to hypertonic and inhibited tissues, dissonant notes, one could say. Hypertonic tissues increase tensional forces acting within local and global networks leading to inappropriate densification of fascial structures, fibrosis and neurovascular fascial adhesions. Inhibited tissues, unable to generate sufficient force to ensure appropriate fascial integrity, lead to excessive compression on neurovascular structures like a dissonant note striking a wrong cord. Site-specific fascia tuning pegs provide appropriate frequency and note specific tension and compression ensuring combined forces operate in an omnidirectional manner resulting in pain free physiology, neurology and motion.The role of muscles in metabolism, physiology, heat production and motion is well described within the scientific literature. Less understood is the local role of myofascial structures providing mechanotransductive forces resulting in fascial expansive responses ensuring appropriate gliding and decompression of neurovascular structures. It is proposed that failure of site-specific fascia tuning pegs results in excessive compression, friction, inflammation, pathology, pain and changes in sensations.

KEY WORDS: Biotensegrity, Fascia, Site specific fascia tuning pegs, Tensegrity, Neuropathy, Dynamic ischemia.

Corresponding Author: John Sharkey MSc, Faculty of Medicine, Dentistry and Clinical Sciences, University of Chester/NTC, 15-16aSt Joseph's Parade, Dorset St, DO7 FR6C, Dublin, Ireland.

E-Mail: john.sharkey@ntc.ie

Access this Article online

Quick Response code

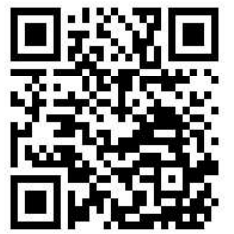

DOI: $10.16965 /$ ijar.2020.254
Journal Information

International Journal of Anatomy and Research

ISSN (E) 2321-4287 | ISSN (P) 2321-8967

https://www.ijmhr.org/ijar.htm

DOI-Prefix: https://dx.doi.org/10.16965/ijar

(cc) ET-No-si

\section{Article Information}

Received: 25 Dec 2020

Peer Review: 25 Dec 2020

Revised: None
Accepted: 27 Jan 2021

Published (O): 20 Feb 2021

Published (P): 05 Mar 2021

\section{INTRODUCTION}

Peripheral neuropathies can have a plethora of origins including physical insults, diabetes, vascular pathology, nutrient issues, hormonal imbalance, kidney and liver pathologies, autoimmune issuances, infections, neurotoxic issues, drug use, tumours and more[1].

Due to the numerus possible aetiologies, appropriate screening and medical history are of paramount importance in ensuring correct diagnosis and effective therapeutic interventions being employed[2]. Magnetic resonance imaging [MRI] can provide essential visual evidence in a complete screening and the overall care of the patient. Site-specific fascia tuning pegs fine-tune the omnipresent fascial net to provide frequency specific tension and compression resulting in stability and energy efficient movement [3]. These combined omnidirectional forces provide finely tuned 
orchestrated motion, physiology and metabolism through the process of mechano transduction [4]. The role that muscles play in metabolism, heat production, physiology and motion is well described within the scientific literature [5]. Less understood is the local role of myofascial structures as they provide mechano transductive forces culminating in a fascial expansion that ensures appropriate gliding and decompression of neurovascular structures [3].

It is proposed that failure of site-specific fascia tuning pegs results in excessive compression, friction, heat, inflammation, pathology, pain and changes in sensations. Tensegrity informed soft-fixed dissections have identified specific structures ideally located to provide local mechano transductive forces hypothe-sised to afford integrity to neurovascular structures providing appropriate tension/ compression [5].

Hypertonic tissues increase tensional forces acting within local, and global, networks. Based on researchfrom Harris, Wild and Stopak (Fig 1) it is hypothesised that inappropriate 'muscle generated forces'can lead to incongruous densification and stiffness of fascial structur essuchas ligaments and aponeurosis [7].

Inappropriate forces may resultin neurovascular fascial adhesions [Fig 2] and fibrosis.

Non-surgical, minimally invasive, fascia focused therapeutic interventions, should be considered in the first instance [3].

German anatomist Julius Wolf demonstrated that loading and unloading of osseofascial tissue [i.e. Wolf's law concerning bone] resulted in specific structural adaptations including bone density [8].In addition, inhibited tissues, unable to generate sufficient mechanical properties andforce sensing in tissues, fail toachieve appropriate shape change and fascial integrity andare proposed to contribute to excessive compression on neurovascular structures [9].

This, in effect, would be similar to a dissonant note striking anerroneouscord. Site-specific fascia tuning pegs are proposedas essential for providingprecise resonance, or note specific tension and compression, ensuring that combined forces operate in an omnidirectional, cooperative manner [3]. This whole body coordination and sharing of forces results in healthy, pain free physiology, neurology, metabolism and motionand is referred to as mechano transduction[10,11]. This is in keeping with Davis's law which describes the phenotypic plasticity of themorphologically softer contributors to our ubiquitous fascial net [14]. Anatomical locations describedas places of perilous passage represent increased risk of insult for nerves as they traverse their path from rootlet to termination [3]. Pain descriptions of lower limb neuropathies include burning, shooting and sharp electrical type pain and deep tingling sensations that impact motor and autonomic activity [8].

Cadaveric dissectionswere performed to identify contractile and non-contractilea natomical structures capable of facilit atingmechanotransductive forces required to reduce compressive forces on site specific neurovascular structures [3].

Several modest myofascial structures, Lacertusfibrosis, psoas minor, psoas tertius/ accessorius, rectus capitis posterior minor, plantaris, pyramidalisare the source of debate concerning their precise function, with many considered vestigial $[16,17,18]$. While a functional description of such modest structures has been attempted it is not satisfying enough to suggest that psoas minor and psoas tertius/accessoriusmaycontribute forces necessary fortrunk flexion.Dissection of the long posterior abdominal wall muscle, psoas minor, highlighted its relationship to the iliopectineal eminence and pelvic fascia located superior to the acetabulum, ideally placed to decompress local and more global neurovascular and lymphatic vessels [3].

For example, rectus capitis posterior minor has been shown to be a muscle rich in proprioceptive nerve endings [13].Its fascial continuityis proposed as a site-specific fascia tuning peg [i.e., myodural bridge] for the upper cervical and occipital dura mater and movement of cerebrospinal fluid [10]. With larger myofascial structures contributing to the cranial base [e.g. upper trapezius], rectus 
capitis posterior minor would offer little in terms of head movement.An alternative, and more satisfying, descriptive role of such modest myofascial constructs is fine tuning fascial tone, reducing compression on associated neurovascular structures [3]. It is proposed that inhibition of such myofascial structures, orhypertension in more distal fascia, results in increased tenson and can resultin inappropriate tension-compression relationships expressed body-wide [19].

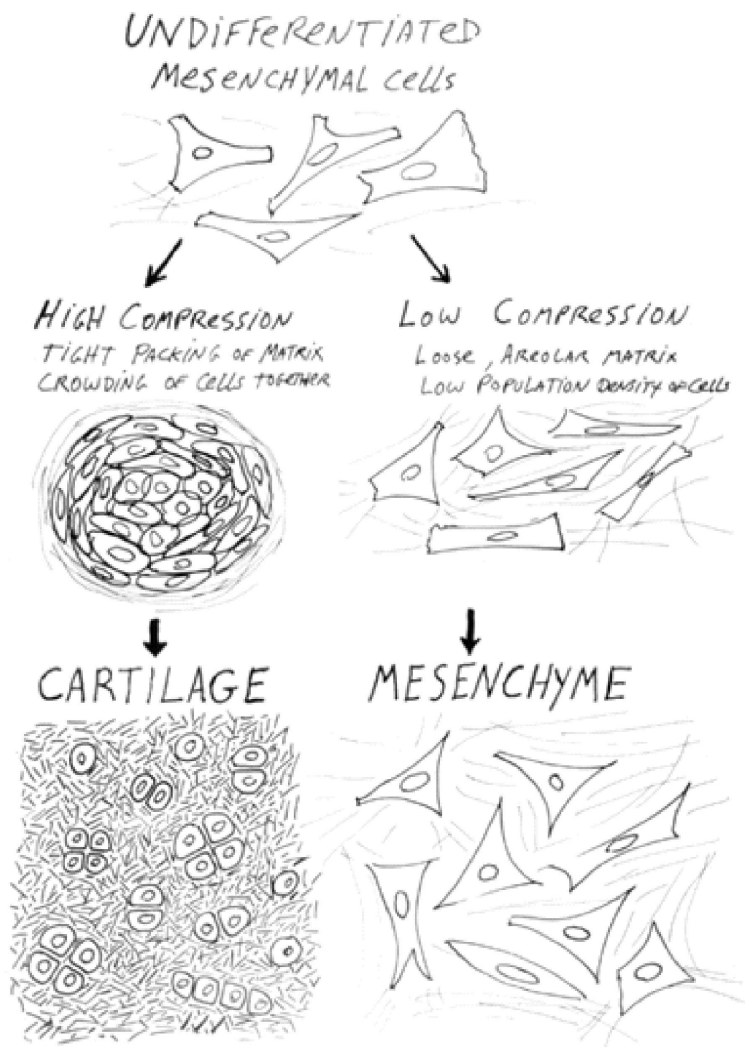

Fig.1: Load distribution, not DNA alone, contributes to the specificity of osseofascial architecture. High compression sites result in tight packing of matrix crowding cells together leading to the production of cartilage. Low compression sites result in loose areolar matrix and low population of density of cells. Image used with permission: Harris, Wild and Stopak, 1980.

Lumbar plexus: The lumbar plexus is prisoner to the fascia of the psoas major anteriorly and quadratus lumborum posteriorlyas the $\mathrm{L} 1$ to L4 roots of ventral rami emerge posterior to the psoas muscle before penetrating its gastor [9]. Nerves emerge placed medial and lateral to the psoas major as follows:

Laterally: iliohypogastric, ilioinguinal, genitofemoral and lateral femoral cutaneous nerve of thigh.

Medial: obturator, lumbosacral trunk.
Dissection of the lumbar plexus confirmed it as a bi-lateral interweaving of six peripheral nerve branchesexisting the T12 to L5 spinal cord levels located in front ofthe corresponding transverse process [3]. The intervertebral foraminal entrance zone represents the earliest peripheral location or place of perilous passage where lumbar nerves can be insulted and/or compressed [20]. Nerve insults to sciatic, pudendal, genitofemoral, obturator, femoral or lateral femoral cutaneous nervescan occur at a number of locations along their course before and after they exit the pelvis due to lack of appropriate tissue gliding [21]. One branch, nerve to obturator, exists through the obturator foramen a possible site of perilous passage. One should consider double or multiple crush compressions culminating in a complex, and possibly, confusing picture during clinical assessment [22]. Intervertebral disk pathology should be ruled out as the primary or contributing cause of patients complaint as well as checking site specific fascia tuning pegs such as psoas minor fascia dysfunction leading to compression of the neurovascular structures such as the genitofemoral nerve. Psoas major fascia is in direct continuity with the endopelvic fascia including the iliolumbar ligament and the fascia overlying muscle iliacus. Fascia integrity, provided by mechanotransductive forces from quadratus femoris and psoas major, are hypothesised to reduce excessive compressional forces on the subcostal nerve, iliohypogastric nerve, ilioinguinal nerve, lateral femoral nerve of thigh and femoral nerve [3].

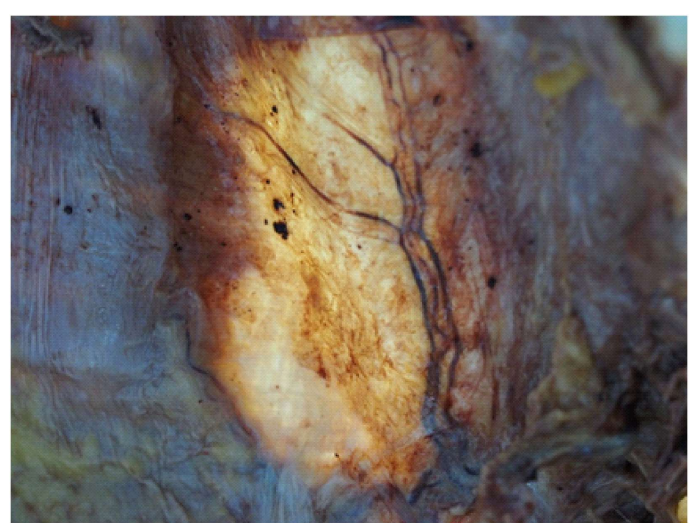

Fig.2:The superficial fascia in continuity with the deep adipose tissue houses the superficial vascular plexus as well as the superficial lymph glands providing omnidirectional force transfer providing the fascia tuning required to ensure vessels do not collapse. 
Sacral and Lumbosacral plexus: Located anterior to the triangular shaped muscle, piriformis, the sacral plexus contributes motor and sensory lumbosacral outflow from the anterior and posterior divisions of anterior rami L4 to S4 nerve roots.An interdigitation of L5 with a small branch contribution from L4, essentially, forms the lumbosacral trunk as it journey's submerged in fascia, along the medial edge of psoas major. The Lumbosacral plexus primarily constitutes the sciatic nerve, combining the common peroneal nerve and the tibial nerve, with the addition of the S4 nerve root. During dissection lumbosacral trunk was trace dinferiorly upon the sacral alacontouring theosseofascial pelvic brim joining the first sacral nerve [3]. The first sacral nerve was seen to be invested in the parietal fascia overlaying piriformis. Innervation of the posterior thigh, portions of the pelvis, the majority of the anatomical leg and the entire foot come from the sciatic, tibial and fibular nerves which are all major branches of the sacral plexus [23].

Biotensegrity focused dissection investigation followed the posterior division as the superior gluteal nerve with innervations clearly identifiable to gluteus medius, gluteus minimus and tensor fascia latae. The inferior gluteal nerve was traced to its terminal point of innervation, the gluteus maximus [3].

Dissection confirms that there are no straight lines in the human body. Bones, nerves, muscle fibers, septal tissues, aponeurosis, blood vessels all exhibit a spiral rotating anatomy. Few tensegrity models reflect this anatomy and therefore most models do not replicate the forces operating in a living tensegrity accurately (Fig3a and 3b). Site specific fascia tuning pegs were identified including the fascia overlying piriformis muscle at the superior portion of the greater sciatic foramen where the superior gluteal nerve, artery andvein twist and wind to exit the pelvis. Failure of the piriformis fascia to provide specific tension in this region is hypothesised to result in compression of these neurovascular structures [3]. Specific divisions of the sciatic nerve include the tibial nerve and its terminal branches. These include the medial and lateral plantar nerves, common fibular nerve [previously known as common peroneal nerve] including its deep and superficial branches. Plantaris is strategically positioned to act as a site specific fascia tuning peg providing expansive forces to neurovascular structures crossing the knee joint.

\section{CONCLUSION}

Lower extremity and pelvic entrapment neuropathies are too often accompanied by confusing clinical manifestations. Such confusion can lead to incorrect diagnosis and therefore inappropriate therapeutic interventions. The model of site specific fascia tuning pegs moves our attention towards a global view in helping to identify proximal ordistal potential sources of insult within a whole-body system. Specific fascial tissues located in strategic anatomical locations are identified as fascia tuning pegs capable of providing the specific resonant force to ensure neurovascular structures are not unduly compressed, tensioned or otherwise insulted.

\section{Conflicts of Interests: None}

\section{REFERENCES}

[1]. Head, K, A. Peripheral neuropathy: pathogenic mechanisms and alternative therapies. Altern Med Rev.2006;11(4):294-329. PMID: 17176168.

[2]. Schleip, R., Gabbiani, G., Wilke, J., Naylor, I., Hinz, B., Zorn, A., Jager, H., Breul, R., Schreiner, S., Klingler, W. Fascia is able to actively contract and may thereby influence musculoskeletal dynamics: A histochemical and mechanographic investigation. Front Physiol. 2019;10:336. doi:10.3389/ fphys.2019.00336.

[3]. Sharkey, J. Site-Specific Fascia Tuning Pegs and Places of Perilous Passage. Myofascia Considerations in Upper Extremity Entrapment Neuropathies -A Clinical Anatomists View. 2020. International Journal of Anatomy and Research. [In Press]

[4]. Romani, P., Valcarcel-Jimenez, L., Frezza, C. et al. Crosstalk between mechanotransduction and metabolism. Nat Rev Mol Cell Biol. 2020.https:// doi.org/10.1038/s41580-020-00306-w

[5]. Sharkey, J. Biotensegrity focused Thiel Soft Fixed Dissection Course. 2019. Department of Anatomy and Human Identification, College of Life Sciences, University of Dundee, Scotland.

[6]. Sharkey J. Tensegrity Informed Observations in Human Cadaveric Studies - A Clinical Anatomists Perspective. Integr J Med Sci. 2020 Oct.15 [cited 2020 Oct.19];7. 
[7]. Harris, A.K., Wild, P., Stopak, D. Silicone rubber substrata: A new wrinkle in the study of cell locomotion. Science1980;208:177-179.

[8]. Frost, H. M. Wolff's Law and bone's structural adaptations to mechanical usage: an overview for clinicians. Angle Orthod. 1994;64:175-88.

[9]. Chanet, S., \& Martin, A. C. Mechanical force sensing in tissues. Progress in molecular biology and translational science. 2014;126:317-352. https:/ /doi.org/10.1016/B978-0-12-394624-9.00013-0

[10]. Ingber, D. Tensegrity-based mechanosensing from macro to micro. Prog Biophys Mol Biol. 2008;97(23):163-179.

[11]. Ingber, D, E. Tensegrity and mechanotransduction. Journal of Bodywork and Movement Therapies. 2008;Jul;12(3):198-200.

[12]. Sharkey, J. Should bone be considered fascia: Proposal for a change in taxonomy of bone- a clinical anatomist's view. International Journal of Biological and Pharmaceutical Sciences Archive. 2020. In press. DOI: 10.30574/ijbpsa

[13]. Zheng N, Yuan XY, Li YF, Chi YY, Gao HB, Zhao X, Yu $S B$, Sui HJ, Sharkey J. Definition of the to be named ligament and vertebrodural ligament and their possible effects on the circulation of CSF. PLoS One. 2014 Aug 1;9[8]:e103451. doi: 10.1371/ journal.pone.0103451. PMID: 25084162; PMCID: PMC4118883.

[14]. Cyron, C. J., \& Humphrey, J. D. Growth and Remodeling of Load-Bearing Biological Soft Tissues. Meccanica, 2017;52(3):645-664. https://doi.org/ 10.1007/s11012-016-0472-5

[15].Martínez Rodríguez, R., \&Galán del Río, F.Mechanistic basis of manual therapy in myofascial injuries. Sonoelastographic evolution control. Journal of bodywork and movement therapies, 2013;17(2):221-234. https://doi.org/ 10.1016/j.jbmt.2012.08.006

[16]. Hallgren, R. C., \& Rowan, J. J. Implied Evidence of the Functional Role of the Rectus Capitis Posterior Muscles. The Journal of the American Osteopathic Association, 2020;120[6]:395-403. https:// doi.org/10.7556/jaoa.2020.061

[17]. Neumann, D. A., \&Garceau, L. R. A proposed novel function of the psoas minor revealed through cadaver dissection. Clinical anatomy [New York, N.Y.], 2015;28(2):243-252. https://doi.org/ $10.1002 /$ ca.22467.
[18]. Olewnik, f., Wysiadecki, G., Podgórski, M., Polguj, M., \&Topol, M. The Plantaris Muscle Tendon and Its Relationship with the Achilles Tendinopathy. BioMed research international, 2018;9623579. https://doi.org/10.1155/2018/9623579.

[19]. Tozzi, P. Selected fascial aspects of osteopathic practice. Journal of bodywork and movement therapies, 2012;16(4):503-519. https://doi.org/10.1016/ j.jbmt.2012.02.003

[20].Tanaka, Nobuhiro., Fujimoto, Yoshinori., An, Howard., Ikuta, Yoshikazu., Yasuda, Mineo. The Anatomic Relation Among the Nerve Roots, Intervertebral Foramina, and Intervertebral Discs of the Cervical Spine. Spine.2000;25:286-91.

[21].Martin, R., Martin, H. D.,Kivlan, B. R. Nerve EntrapmentIn The Hip Region: Current Concepts Review. International journal of sports physical therapy, 2017;12(7):1163-1173. https://doi.org/ $10.26603 /$ ijspt20171163.

[22]. Ang, Chia-Liang., Foo, Leon. Multiple Locations of Nerve Compression: An Unusual Cause of Persistent Lower Limb Paresthesia. The Journal of foot and ankle surgery: official publication of the American College of Foot and Ankle Surgeons. 2014;53. 10.1053/j.jfas.2014.06.013.

[23]. Singh O, Al Khalili Y. Anatomy, Back, Lumbar Plexus. [Updated 2020 Aug 10]. In: StatPearls [Internet]. Treasure Island (FL): StatPearls Publishing; 2020 Jan-. Available from: https://www.ncbi.nlm.nih.gov/books/NBK545137/.

[24]. McPartland, M, John., Brodeur, R, Raymond. Rectus capitis posterior minor: a small but important suboccipital muscle,Journal of Bodywork and Movement Therapies, 1999;3(1):30-35.

How to cite this article:

John Sharkey. Fascia and living tensegrity considerations in lower extremity and pelvic entrapment neuropathies. Int J Anat Res 2021;9(1.2):7881-7885. DOI: 10.16965/ijar.2020.254 